\title{
A Multiplication Formula for the Modified Caldero-Chapoton Map
}

\author{
David Pescod ${ }^{1}$
}

Received: 6 October 2016 / Accepted: 29 July 2018 / Published online: 9 August 2018

(c) The Author(s) 2018

\begin{abstract}
A frieze in the modern sense is a map from the set of objects of a triangulated category C to some ring. A frieze $X$ is characterised by the property that if $\tau x \rightarrow y \rightarrow x$ is an Auslander-Reiten triangle in $\mathrm{C}$, then $X(\tau x) X(x)-X(y)=1$. The canonical example of a frieze is the (original) Caldero-Chapoton map, which send objects of cluster categories to elements of cluster algebras. Holm and Jørgensen (Nagoya Math J 218:101-124, 2015; Bull Sci Math 140:112-131, 2016), the notion of generalised friezes is introduced. A generalised frieze $X^{\prime}$ has the more general property that $X^{\prime}(\tau x) X^{\prime}(x)-X^{\prime}(y) \in\{0,1\}$. The canonical example of a generalised frieze is the modified Caldero-Chapoton map, also introduced in Holm and Jørgensen (2015, 2016). Here, we develop and add to the results in Holm and Jørgensen (2016). We define Condition $\mathrm{F}$ for two maps $\alpha$ and $\beta$ in the modified CalderoChapoton map, and in the case when $C$ is 2-Calabi-Yau, we show that it is sufficient to replace a more technical "frieze-like" condition from Holm and Jørgensen (2016). We also prove a multiplication formula for the modified Caldero-Chapoton map, which significantly simplifies its computation in practice.
\end{abstract}

Keywords Auslander-Reiten triangle · Categorification · Cluster algebra $\cdot$ Cluster category $\cdot$ Cluster tilting object $\cdot$ Cluster tilting subcategory $\cdot$ Rigid object $\cdot$ Rigid subcategory $\cdot$ Triangulated category

Mathematics Subject Classification 05E10 - 13F60 - 16G70 - 18E30

Communicated by M. M. Clementino.

$凶 \quad$ David Pescod

d.pescod1@ncl.ac.uk

1 School of Mathematics and Statistics,Newcastle University, Newcastle Upon Tyne NE1 7RU, UK 


\section{Introduction}

\subsection{Summary}

This paper focuses around two main topics: generalised friezes with integer values (see [14]) and generalised friezes taking values inside a Laurent polynomial ring (see [15]).

A frieze is a map $X: \operatorname{objC} \rightarrow A$, where $C$ is some triangulated category with AuslanderReiten (AR) triangles and $A$ is a ring, such that the following exponential conditions are satisfied:

$$
X(0)=1 \text { and } X(a \oplus b)=X(a) X(b),
$$

and if $\tau x \rightarrow y \rightarrow x$ is an AR triangle, then

$$
X(\tau x) X(x)-X(y)=1 .
$$

The canonical example of a frieze is the Caldero-Chapoton map, which we recall in Sect. 1.4.

Generalised friezes are similarly defined maps $X^{\prime}:$ objC $\rightarrow A$, also satisfying the exponential conditions in (1.1), however we permit the more general property that

$$
X^{\prime}(\tau x) X^{\prime}(x)-X^{\prime}(y) \in\{0,1\} .
$$

The canonical example of a generalised frieze is the modified Caldero-Chapoton map, which we recall in Sect. 1.6. The arithmetic version $\pi$, with integer values, is defined in Eq. (1.5), whilst the more general version $\rho$, taking values inside a Laurent polynomial ring, is defined in Eq. (1.8).

The modified Caldero-Chapoton map was introduced in [15], and we improve and add to the results of that paper. When working with a 2-Calabi-Yau category, we manage to replace the technical "frieze-like" condition (see [15, def. 1.4]) for the maps $\alpha$ and $\beta$ in the generalised Caldero-Chapoton map (Eq. 1.8), by our so-called Condition F (see Definition 3.1). This condition significantly simplifies the frieze-like condition and demonstrates the roles of $\alpha$ and $\beta$. We will see that $\alpha$ plays the role of a "generalised index", whilst $\beta$ provides a correction term to $\alpha$ being exponential over a distinguised triangle.

We use this to establish a multiplication formula for the modified Caldero-Chapoton map $\rho$ (see Theorem 6.2), allowing its computation in practice. In [15], the computation of $\rho$ is not addressed. However, our multiplication formula does address the computation, and does so in a simpler manner than merely applying the definition. In particular, the formula allows us to compute values of $\rho$ without calculating Euler characteristics of submodule Grassmannians which are otherwise part of the definition of $\rho$.

\subsection{Cluster Categories}

Cluster categories were first introduced by Buan et al. [4] as a means of understanding the 'decorated quiver representations' introduced by Reineke et al. [16]. Let $Q$ be a finite quiver with no loops or cycles, and consider the category $\bmod \mathbb{C} Q$ of finitely generated modules over the path algebra $\mathbb{C} Q$. Then, set

$$
\mathrm{D}(Q)=\mathrm{D}^{b}(\bmod \mathbb{C} Q),
$$

the bounded derived category of $\bmod \mathbb{C} Q$.

The cluster category of type $Q$, denoted $\mathrm{C}(Q)$, is defined to be the orbit category of $\mathrm{D}(Q)$ under the action of the cyclic group generated by the autoequivalence $\tau^{-1} \Sigma=S^{-1} \Sigma^{2}$, where 
$\tau$ is the Auslander-Reiten translation, $\Sigma$ the suspension functor and $S$ the Serre functor. That is,

$$
\mathrm{C}(Q)=\mathrm{D}(Q) /\left(S^{-1} \Sigma^{2}\right)
$$

The objects in $\mathrm{C}(Q)$ are the same as those in $\mathrm{D}(Q)$, however, the morphism sets in $\mathrm{C}(Q)$ are given by

$$
\operatorname{Hom}_{C(Q)}(X, Y)=\bigoplus_{n \in \mathbb{Z}} \operatorname{Hom}_{\mathrm{D}(Q)}\left(X,\left(S^{-1} \Sigma^{2}\right)^{n} Y\right)
$$

We note that $\mathrm{C}(Q)$ possesses a triangulated structure, it is $\mathbb{C}$-linear, Hom-finite, Krull-Schmidt and 2-Calabi-Yau, meaning that its Serre functor is $\Sigma^{2}$. It is also essentially small and has split idempotents. See [4, sec. 1]

The cluster category of Dynkin type $A_{n}$, denoted by $\mathrm{C}\left(A_{n}\right)$, has a very nice polygon model associated to it. This is due to Caldero et al. [7], who defined, for finite quivers of type $A_{n}$, an equivalent category to the cluster category in [4] in a totally different manner. This is done using a triangulation of a regular $(n+3)$-gon $P$, with objects and morphisms described in [7, sec. 2].

The category $\mathrm{C}\left(A_{n}\right)$ carries several nice properties. There is a bijection between the set of indecomposables of $\mathrm{C}\left(A_{n}\right)$, denoted indec $\mathrm{C}\left(A_{n}\right)$, and the set of diagonals of $P$. We also identify each edge of $P$ with the zero object in $\mathrm{C}\left(A_{n}\right)$. Applying the suspension functor $\Sigma$ to an indecomposable corresponds to rotating the endpoints of the associated diagonal one vertex clockwise. That is, if the vertices of $P$ are labelled in an anticlockwise fashion with the set $\{1,2, \ldots, n\}$, then for some indecomposable $\{i, j\}$, where $i$ and $j$ are vertices of $P$, we have

$$
\Sigma\{i, j\}=\{i-1, j-1\} .
$$

Such coordinates should clearly be taken modulo $n+3$.

Identifying indecomposables of $\mathrm{C}\left(A_{n}\right)$ with the diagonals of $P$ carries the convenient property that for $a, b \in \operatorname{indec} C\left(A_{n}\right)$,

$$
\operatorname{dim}_{\mathbb{C}} \operatorname{Ext}_{C\left(A_{n}\right)}^{1}(a, b)=\left\{\begin{array}{l}
1, \text { if } a \text { and } b \text { cross } \\
0, \text { if } a \text { and } b \text { do not cross. }
\end{array}\right.
$$

\subsection{The Auslander-Reiten Quiver}

The Auslander-Reiten quiver for $\mathrm{C}\left(A_{n}\right)$ is $\mathbb{Z} A_{n}$ modulo a glide reflection. A coordinate system may be put on the quiver, matching up with the diagonals of the $(n+3)$-gon [see Fig. 1 for an example of $\left.C\left(A_{5}\right)\right]$.

The Auslander-Reiten triangles in $\mathrm{C}\left(A_{n}\right)$ take the form

$$
\{i-1, j-1\} \rightarrow\{i-1, j\} \oplus\{i, j-1\} \rightarrow\{i, j\},
$$

where if $\{i-1, j\}$ or $\{i, j-1\}$ correspond to an edge of $P$, then they should be taken to be zero, see [5, lem. 3.15]. Notice that each AR triangle can be realised from a diamond in the AR quiver. That is, if 


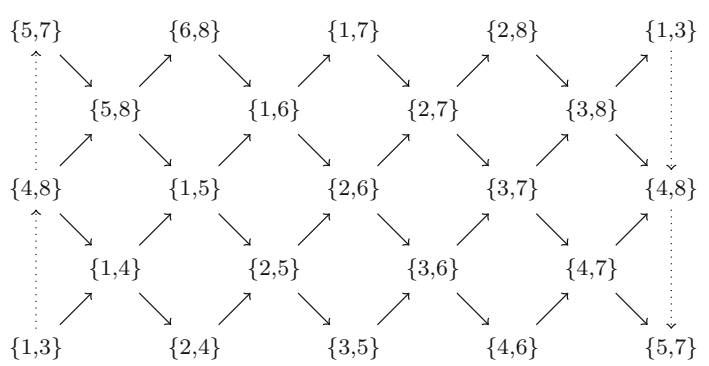

Fig. 1 The Auslander-Reiten quiver for $\mathrm{C}\left(A_{5}\right)$. The dotted lines are identified with opposite orientations

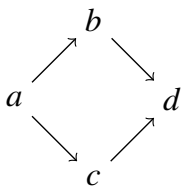

is a diamond inside the AR quiver, then

$$
a \rightarrow b \oplus c \rightarrow d
$$

is an AR triangle. If $a$ and $d$ sit on the upper boundary of the AR quiver, then $b$ should be taken as zero, whereas if $a$ and $d$ sit on the lower boundary, then $c$ is taken to be zero. Note that a frieze $X$ on $C\left(A_{n}\right)$ satisfies:

$$
X(a) X(d)-X(b) X(c)=1 .
$$

\subsection{The Caldero-Chapoton Map}

The (original) Caldero-Chapoton map is a map which sends certain (so-called reachable) indecomposable objects of a cluster category to cluster variables of the corresponding cluster algebra, see [8, sec. 4.1]. The map, which we denote by $\gamma$, depends on a cluster tilting object $T$ inside the cluster category and makes precise the idea that the cluster category is a categorification of the cluster algebra. It is required that the category is 2-Calabi-Yau (for example a cluster category), and it is a well known property of $\gamma$ that it is a frieze (see [1, def. 1.1], [6, prop. 3.10], [12, thm.]). That is, the Caldero-Chapoton map is a map $\gamma: \operatorname{obj} C \rightarrow A$, where $A$ is a certain ring, which satisfies the property in Eq. (1.2), as well as the exponential conditions in (1.1).

\subsection{Frieze Patterns}

Frieze patterns were first introduced by Conway and Coxeter $[9,10]$. An example of such a frieze pattern, known as a Conway-Coxeter frieze, is given in Fig. 2. This frieze pattern is obtained from the original Caldero-Chapoton map $\gamma$ by omitting the arrows from the AR quiver of $\mathrm{C}\left(A_{7}\right)$ and replacing each vertex by the value of $\gamma$ applied to that indecomposable.

In formal terms, for some positive integer $n$, a frieze pattern is an array of $n$ offset rows of positive integers. Each diamond 


$\begin{array}{ccccccccccc}\hat{4} & & 4 & & 1 & & 2 & & 2 & & 4 \\ \hat{\vdots} & 15 & & 3 & & 1 & & 3 & & 7 & \vdots \\ \hat{11} & & 11 & & 2 & & 1 & & 10 & & \vdots \\ \hat{\vdots} & 8 & & 7 & & 2 & & 3 & & 7 & \vdots \\ \vdots & & & & & & & & & \\ 5 & & 5 & & 3 & & 2 & & 2 & & 11 \\ \hat{\vdots} & 3 & & 2 & & 5 & & 1 & & 3 & \vdots \\ \vdots & & & & & & & & & \\ 4 & & 1 & & 3 & & 2 & & 1 & & 4\end{array}$

Fig. 2 A frieze on the cluster category of Dynkin type $A_{7}$. The dotted lines are identified with opposite orientations

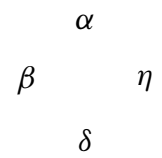

in a frieze pattern satisfies a so-called determinant property in that

$$
\beta \eta-\alpha \delta=1 .
$$

If $\beta$ and $\eta$ are on the top row of the frieze, then the determinant property becomes

$$
\beta \eta-\delta=1,
$$

and if $\beta$ and $\eta$ are on the bottom row, the determinant property becomes

$$
\beta \eta-\alpha=1 .
$$

Observe that the Caldero-Chapoton map satisfies these equations by virtue of Eq. (1.4), that is, because it is a Conway-Coxeter frieze. Conway-Coxeter frieze patterns are known to be invariant under a glide reflection. In this case, a region of the frieze pattern, known as a fundamental domain, is enough to produce the whole frieze pattern by repeatedly performing a glide reflection.

\subsection{A Modified Caldero-Chapoton Map}

We assume in the rest of the paper that $\mathbb{C}$ is an essentially small, $\mathbb{C}$-linear, Hom-finite, triangulated category, which is Krull-Schmidt and has AR triangles. Holm and Jørgensen [14] introduce a modified version of the Caldero-Chapoton map, which we denote by $\pi$, that relies on a rigid object $R \in \operatorname{obj} C$, a much weaker condition than that of being a cluster tilting object. We say that an object $R$ is rigid if

$$
\operatorname{Hom}_{C}(R, \Sigma R)=0 .
$$

We also note that $\pi$ does not require that the category is 2-Calabi-Yau, allowing us to work with a category $\mathrm{C}$ that is more general than a cluster category. 
Consider the endomorphism ring $E=\operatorname{End}_{\mathrm{C}}(R)$, and define mod $E$ to be the category of finite dimensional right $E$-modules. Then, there is a functor

$$
\begin{aligned}
G: C \rightarrow \bmod E & \\
c & \mapsto \operatorname{Hom}_{C}(R, \Sigma c) .
\end{aligned}
$$

For some object $c \in \mathrm{C}$, the modified Caldero-Chapoton map is then defined by the formula:

$$
\pi(c)=\chi(\operatorname{Gr}(G c)),
$$

where Gr denotes the Grassmannian of submodules and $\chi$ is the Euler characteristic defined by cohomology with compact support (see [13, p. 93]).

It is proved in [14] that $\pi$ is a generalised frieze; that is, as well as the exponential properties given in Eq. (1.1) it satisfies the property given in Eq. (1.3).

Define $\mathrm{R}=$ add $R$, the full subcategory whose objects are finite direct sums of the summands of $R$ (see Sect. 2 for details of this setup). This full subcategory, which is clearly closed under direct sums and summands, is rigid in the sense that $\operatorname{Hom}_{C}(R, \Sigma R)=0$. A multiplication formula for computing $\pi$ is also proved in [14]. Let $m \in$ indec $C$ and $r \in$ indec R satisfy that $\operatorname{Ext}_{C}^{1}(m, r)$ and $\operatorname{Ext}_{C}^{1}(r, m)$ both have dimension one over $\mathbb{C}$. Then, there are nonsplit triangles

$$
m \stackrel{\mu}{\longrightarrow} a \stackrel{\gamma}{\longrightarrow} r \stackrel{\delta}{\longrightarrow} \Sigma m, r \stackrel{\sigma}{\longrightarrow} b \stackrel{\eta}{\longrightarrow} m \stackrel{\zeta}{\longrightarrow} \Sigma r
$$

that are unique up to isomorphism. It is proved in [14] that

$$
\pi(m)=\pi(a)+\pi(b) .
$$

This formula can be applied iteratively to compute values of $\pi$.

Holm and Jørgensen [15] redefine the modified Caldero-Chapoton map in a more general manner (the work in [14] is a special case of that in [15]). They define $\rho$ by

$$
\rho(c)=\alpha(c) \sum_{e} \chi\left(\operatorname{Gr}_{e}(G c)\right) \beta(e) .
$$

Here the sum is taken over $e \in \mathrm{K}_{0}(\bmod E)$, the Grothendieck group of the abelian category $\bmod E$, and $\mathrm{Gr}_{e}(G c)$ is the Grassmannian of $E$-submodules $M \subseteq G c$ with $\mathrm{K}_{0}$-class satisfying $[M]=e$. The maps

$$
\alpha: \operatorname{obj} C \rightarrow A \text { and } \beta: \mathrm{K}_{0}(\bmod E) \rightarrow A
$$

are both exponential maps in the sense that

$$
\begin{aligned}
& \alpha(0)=1, \alpha(x \oplus y)=\alpha(x) \alpha(y), \\
& \beta(0)=1, \beta(e+f)=\beta(e) \beta(f),
\end{aligned}
$$

and $A$ is some commutative ring, see [15, setup 1.1].

When the maps $\alpha$ and $\beta$ satisfy a technical "frieze-like" condition, given in [15, def. 1.4], the map $\rho$ becomes a generalised frieze, as in Eq. (1.3).

\subsection{This Paper}

In this paper, we show a simpler condition on $\alpha$ and $\beta$ than that in [15], which implies that $\rho$ is a generalised frieze. We also show that a similar multiplication formula to that in Eq. (1.7) 
holds with $\rho$ instead of $\pi$. This permits a simpler iterative procedure for computing $\rho$ than the one given in [15].

We give the following definition:

Definition 3.1 (Condition $F$ ) We say that the maps $\alpha$ and $\beta$ satisfy Condition $\mathrm{F}$ if for each triangle

$$
x \stackrel{\varphi}{\longrightarrow} y \stackrel{\omega}{\longrightarrow} z \stackrel{\psi}{\longrightarrow} \Sigma x
$$

in C such that $G x, G y$ and $G z$ have finite length in $\operatorname{Mod} E$, the following property holds:

$$
\alpha(y)=\alpha(x \oplus z) \beta([\operatorname{Ker} G \varphi]) .
$$

The following main result shows that when $\mathrm{C}$ is 2-Calabi-Yau, this definition is sufficient to replace the frieze-like condition from [15, def. 1.4].

Theorem 3.2 Assume that the exponential maps $\alpha: \operatorname{obj} C \rightarrow A$ and $\beta: K_{0}(\bmod E) \rightarrow A$ from Eq. (1.9) satisfy Condition F from Definition 3.1. Then, the modified Caldero-Chapoton map $\rho$ of Eq. (1.8) is a generalised frieze in the sense that it satisfies Eq. (1.3), as well as the exponential conditions in (1.1).

We then proceed by proving in Lemma 4.1 that the construction of $\alpha$ and $\beta$ in [15, def. 2.8] satisfies Condition F. Then, Theorem 3.2, together with Lemma 4.1, recovers a main result of [15], proving that the construction of $\alpha$ and $\beta$ in [15, def. 2.8] results in $\rho$ being a generalised frieze.

In Sect. 6, we prove the multiplication formula for $\rho$, similar to the arithmetic case for $\pi$ in (1.7). This multiplication formula is as follows:

Theorem 6.2 Let $m \in \operatorname{indec} C$ and $r \in \operatorname{indec} R$ such that $\operatorname{Ext}_{C}^{1}(r, m)$ and $\operatorname{Ext}_{C}^{1}(m, r)$ both have dimension one over $\mathbb{C}$. Then, there are nonsplit triangles

$$
m \stackrel{\mu}{\longrightarrow} a \stackrel{\gamma}{\longrightarrow} r \stackrel{\delta}{\longrightarrow} \Sigma m \quad \text { and } \quad r \stackrel{\sigma}{\longrightarrow} b \stackrel{\eta}{\longrightarrow} m \stackrel{\zeta}{\longrightarrow} \Sigma r,
$$

with $\delta$ and $\zeta$ nonzero. Let Gm have finite length in ModE, then

$$
\rho(r) \rho(m)=\rho(a)+\rho(b) .
$$

This theorem can be applied inductively in order to simplify the computation of values of $\rho$. For some indecomposable $m$ in C, one may find an $a$ and $b$ (which are the middle terms of the nonsplit extensions), such that $\rho(m)=\rho(a)+\rho(b)$. The theorem can then be reapplied to find each of $\rho(a)$ and $\rho(b)$. The process will eventually terminate at the stage where calculating $\rho$ of some indecomposable reduces to calculating the index of that indecomposable. Substituting back into the equation allows a simple calculation of $\rho(m)$.

We illustrate the procedure in Sect. 7 by computing $\rho$ of an indecomposable in the Auslander-Reiten quiver for $C\left(A_{5}\right)$. This retrieves one of the vertices of the AR quiver in Fig. 3. Note that this example already appeared in [15, sec. 3], but Theorem 6.2 makes our computation much simpler.

This paper is organised as follows. Section 2 gives an essential background to the modified Caldero-Chapoton map, and explains some important results from [15]. Section 3 introduces Condition F and proves Theorem 3.2, whilst Sect. 4 shows how one can construct maps $\alpha$ and $\beta$ satisfying this condition. Section 5 demonstrates how to find the multiplication formula 


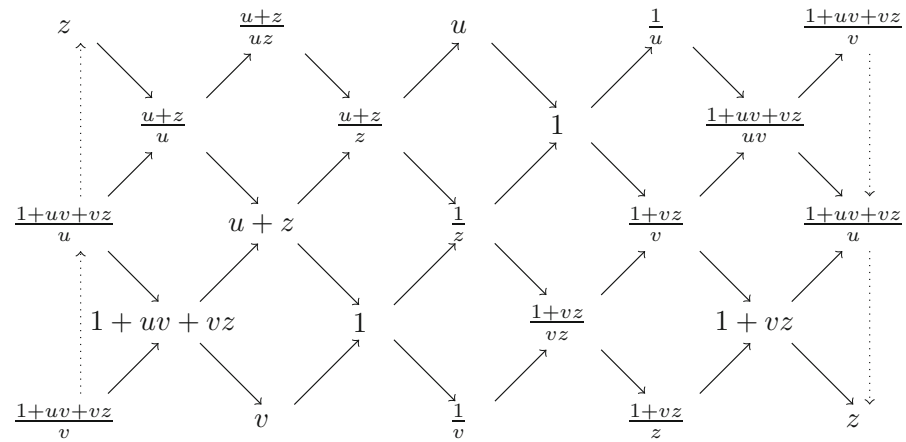

Fig. 3 The Auslander-Reiten quiver of the cluster category of Dynkin type $A_{5}$. The vertices have been replaced with values of the modified Caldero-Chapoton map $\rho$. Again, the dotted lines are identified with oppostite orientations

for $\pi$, Sect. 6 adapts this formula to $\rho$ by proving the multiplication formula in Theorem 6.2 , and Sect. 7 shows why this formula is useful.

It should be noted that Sects. 2 and 5 contain no original work, however they provide an essential setup for subsequent sections in the paper.

\section{A Modified Caldero-Chapoton Map: A Functorial Viewpoint}

In this section, we redefine the modified Caldero-Chapoton map in detail, using an equivalent, functorial viewpoint, allowing us simpler calculations throughout the rest of the paper. To set up, we will follow the construction in [15, sec. 2]. We add the assumption that $C$ is 2Calabi-Yau. We let $R$ be a functorially finite subcategory of $C$, which is closed under sums and summands and rigid; that is, $\operatorname{Hom}_{\mathrm{C}}(\mathrm{R}, \Sigma \mathrm{R})=0$.

We also assume that $C$ has a cluster tilting subcategory $T$, belonging to a cluster structure in the sense of [3, sec. II.1]. We additionally require that $\mathrm{R} \subseteq \mathrm{T}$. Note that the Auslander-Reiten translation for $\mathrm{C}$ is

$$
\tau=\Sigma,
$$

and its Serre functor is $S=\Sigma^{2}$.

There is a functor $G$, defined by:

$$
\begin{aligned}
G: \mathrm{C} & \rightarrow \operatorname{ModR} \\
c & \left.\mapsto \operatorname{Hom}_{\mathrm{C}}(-, \Sigma c)\right|_{\mathrm{R}}
\end{aligned}
$$

where Mod $R$ denotes the category of $\mathbb{C}$-linear contravariant functors $R \rightarrow$ Vect $\mathbb{C}$. It is a $\mathbb{C}$-linear abelian category, and we denote by fl R the full subcategory of Mod R, consisting of all finite length objects.

The modified Caldero-Chapoton map is then defined as in Eq. (1.8), where the sum is now taken over $e \in \mathrm{K}_{0}$ (fl $\left.\mathrm{R}\right)$. Here, we have

$$
\alpha: \operatorname{obj} C \rightarrow A, \beta: \mathrm{K}_{0}(\mathrm{flR}) \rightarrow A,
$$

and these maps satisfy the exponential condition in Eq. (1.10) 
For two objects $a, b \in \mathrm{C}$ such that $G a$ and $G b$ have finite length, it is known by [15, prop. 1.3] that $\rho$ is also exponential; that is,

$$
\rho(0)=1, \rho(a \oplus b)=\rho(a) \rho(b) .
$$

It therefore suffices to calculate $\rho$ for each indecomposable object in C. Note that the formula for $\rho$ only makes sense when $G c$ has finite length in Mod R. That is, we require that $G c \in \mathrm{flR}$.

\section{Condition F on the Maps $\alpha$ and $\beta$}

We continue under the setup of Sect. 2. Consider the exponential maps $\alpha$ and $\beta$ introduced earlier in Eq. (2.2). The following definition describes a canonical condition on $\alpha$ and $\beta$, which will later be used in Sect. 6 to prove a multiplication formula for $\rho$.

Definition 3.1 (Condition $F$ ) We say that the maps $\alpha$ and $\beta$ satisfy Condition $\mathrm{F}$ if, for each triangle

$$
x \stackrel{\varphi}{\longrightarrow} y \stackrel{\omega}{\longrightarrow} z \stackrel{\psi}{\longrightarrow} \Sigma x
$$

in C, such that $G x, G y$ and $G z$ have finite length in Mod R, the following property holds:

$$
\alpha(y)=\alpha(x \oplus z) \beta([\operatorname{Ker} G \varphi]) .
$$

We now prove a theorem showing that in the case when $\mathrm{C}$ is 2-Calabi-Yau, Condition $\mathrm{F}$ can replace the definition of the frieze-like condition defined in [15, def. 1.4].

Theorem 3.2 Assume that the exponential maps $\alpha: \operatorname{obj} C \rightarrow A$ and $\beta: K_{0}(f l R) \rightarrow A$ from $E q$. (2.2) satisfy Condition F from Definition 3.1. Then, the modified Caldero-Chapoton map $\rho$, defined in Eq. (1.8), is a generalised frieze in the sense that, where defined, it satisfies Eq. (1.3), as well as the exponential conditions in (1.1).

Proof Note that by $[15$, thm. 1.6$]$, if

$$
\Delta=(\Sigma c \stackrel{\xi}{\longrightarrow} b \longrightarrow c)
$$

is an AR triangle in $C$ such that $G c$ and $G \Sigma c$ have finite length in Mod R, and $\alpha$ and $\beta$ are frieze-like maps for $\Delta$ in the sense of [15, def. 1.4], then $\rho(\Sigma c) \rho(c)-\rho(b) \in\{0,1\}$. Hence, it suffices to show that $\alpha$ and $\beta$ are frieze-like for each $\Delta$. We first note that if $c \notin \mathrm{R} \cup \Sigma^{-1} \mathrm{R}$, then $G(\Delta)$ is a short exact sequence, see [14, lem. 1.12(iii)]. We now check the three cases of [15, def. 1.4].

Case (i) Assume $c \notin \mathrm{R} \cup \Sigma^{-1} \mathrm{R}$ and $G(\Delta)$ is a split short exact sequence. That is,

$$
0 \longrightarrow G(\Sigma c) \stackrel{G \xi}{\longrightarrow} G b \longrightarrow G c \longrightarrow 0,
$$

is split short exact. It follows immediately that $G \xi$ has trivial kernel. Applying Condition $\mathrm{F}$ to $\Delta$, we obtain:

$$
\begin{aligned}
\alpha(b) & =\alpha(c \oplus \Sigma c) \beta([\operatorname{Ker} G \xi]) \\
& =\alpha(c \oplus \Sigma c) \beta(0) \\
& =\alpha(c \oplus \Sigma c)
\end{aligned}
$$

where the final $=$ is due to $\beta$ being exponential. 
Case (ii) first part Assume $c \notin \mathrm{R} \cup \Sigma^{-1} \mathrm{R}$ and $G(\Delta)$ is a nonsplit short exact sequence. Then, by the same working as in Case (i), we see that:

$$
\alpha(b)=\alpha(c \oplus \Sigma c) .
$$

Now, consider the following triangle in C:

$$
c \stackrel{v}{\longrightarrow} 0 \longrightarrow \Sigma c \stackrel{1_{\Sigma c}}{\longrightarrow} \Sigma c .
$$

Applying Condition F and using the fact that $\alpha$ is exponential, we obtain:

$$
\begin{aligned}
1 & =\alpha(0) \\
& =\alpha(c \oplus \Sigma c) \beta([\operatorname{Ker} G \nu]) \\
& =\alpha(c \oplus \Sigma c) \beta([G c]) .
\end{aligned}
$$

We note that this manipulation works for any $c \in \operatorname{obj} C$.

Case (ii) second part Let $c=\Sigma^{-1} r \in \Sigma^{-1} \mathrm{R}$. We showed in the first part of Case (ii) that

$$
\alpha(c \oplus \Sigma c) \beta([G c])=1 .
$$

Now, by [14, lem. 1.12(i)], $G(\Delta)$ becomes:

$$
G(\Delta)=0 \rightarrow \operatorname{rad} P_{r} \rightarrow P_{r},
$$

where $P_{r}$ is the indecomposable projective $\operatorname{Hom}_{\mathrm{R}}(-, r)$ in Mod R, described in [14, sec. 1.5]. Therefore, $G \xi$ has zero kernel, and applyng Condition F shows:

$$
\begin{aligned}
\alpha(b) & =\alpha(c \oplus \Sigma c) \beta([\operatorname{Ker} G \xi]) \\
& =\alpha(c \oplus \Sigma c) \beta(0) \\
& =\alpha(c \oplus \Sigma c) .
\end{aligned}
$$

Case (iii) Let $c=r \in \mathrm{R}$ and again consider the triangle

$$
c \stackrel{v}{\longrightarrow} 0 \longrightarrow \Sigma c \stackrel{1 \Sigma c}{\longrightarrow} \Sigma c
$$

in C. Applying Condition F, we see

$$
\begin{aligned}
1 & =\alpha(0) \\
& =\alpha(c \oplus \Sigma c) \beta([\operatorname{Ker} G v]) \\
& =\alpha(c \oplus \Sigma c) \beta(0) \\
& =\alpha(c \oplus \Sigma c),
\end{aligned}
$$

where the third $=$ is since $G(c)=G(r)=0$ and hence $\operatorname{Ker} G v=0$.

Now, by [14, lem. 1.12(ii)], applying $G$ to $\Delta$ gives the exact sequence:

$$
I_{r} \stackrel{G \xi}{\longrightarrow} \operatorname{corad} I_{r} \longrightarrow 0,
$$

where $I_{r}$ is the indecomposable injective $\operatorname{Hom}_{\mathrm{R}}\left(-, \Sigma^{2} r\right)$ in Mod R, described in [14, sec. 1.10] and corad $I_{r}$ is its coradical. Additionally, by [14, sec. 1.10], we know that there is the following short exact sequence:

$$
0 \rightarrow S_{r} \rightarrow I_{r} \rightarrow \operatorname{corad} I_{r} \rightarrow 0,
$$


where $S_{r}$ in Mod R is the simple object supported at $r$, see [2, prop. 2.2]. It follows that corad $I_{r} \cong I_{r} / S_{r}$ and we see that $\operatorname{Ker} G \xi=S_{r}$. So, by applying Condition F once again, we see that

$$
\begin{aligned}
\alpha(b) & =\alpha(c \oplus \Sigma c) \beta([\operatorname{Ker} G \xi]) \\
& =\beta([\operatorname{Ker} G \xi]) \\
& =\beta\left(\left[S_{r}\right]\right),
\end{aligned}
$$

where the second $=$ is since $\alpha(c \oplus \Sigma c)=1$.

\section{Constructing Maps that Satisfy Condition F}

We again continue under the setup of Sect. 2. We will show that there exist maps $\alpha$ and $\beta$ satisfying Condition F, namely those given in [15, def. 2.8]. Let us first look at the necessary constructions behind the definitions of $\alpha$ and $\beta$ in [15, def. 2.8].

Recall that $\mathrm{T}$ is some cluster tilting subcategory of $\mathrm{C}$ with $\mathrm{R} \subseteq \mathrm{T}$, and denote by indec $\mathrm{T}$ the set of indecomposable objects in T. For each $t \in$ indec $\mathrm{T}$, one may find a unique indecomposable $t^{*} \in$ indec $\mathrm{C}$, called the mutation of $t$, such that replacing $t$ with $t^{*}$ gives rise to another cluster tilting subcategory $\mathrm{T}^{*}$, see [3, sec. II.1]. Each such $t$ and $t^{*}$ fit into two exchange triangles (see [3, sec. II.1]):

$$
t^{*} \rightarrow a \rightarrow t, t \rightarrow a^{\prime} \rightarrow t^{*},
$$

where $a, a^{\prime} \in$ add $((\operatorname{indec} \mathrm{T}) \backslash t)$.

We denote by $K_{0}^{\text {split }}(T)$ the split Grothendieck group of the additive category $T$ which has a basis formed by the set of indecomposables in $\mathrm{T}$. We note that $\mathrm{K}_{0}^{\text {split }}(\mathrm{T})$ carries the relations that $[a \oplus b]=[a]+[b]$, where $[a]$ is used to denote the $\mathrm{K}_{0}^{\text {split }}$-class of the object $a$ in $\mathrm{T}$.

Define $S$ to be the full subcategory of $C$ which is closed under direct sums and summands and has

$$
\text { indec } \mathrm{S}=\text { indec } \mathrm{T} \backslash \text { indec } \mathrm{R},
$$

and then consider the subgroup

$$
N=\left\langle[a]-\left[a^{\prime}\right] \mid \begin{array}{l}
s^{*} \rightarrow a \rightarrow s, s \rightarrow a^{\prime} \rightarrow s^{*} \text { are exchange } \\
\text { triangles with } s \in \text { indec } S
\end{array}\right\rangle
$$

of $\mathrm{K}_{0}^{\text {split }}(\mathrm{T})$, defined in [15, def. 2.4]. Then, we denote by $Q$ the canonical surjection

$$
Q: \mathrm{K}_{0}^{\text {split }}(\mathrm{T}) \rightarrow \mathrm{K}_{0}^{\text {split }}(\mathrm{T}) / N, Q([t])=[t]+N .
$$

Now, for each $c \in \operatorname{obj} C$, we may construct the element $\operatorname{ind}_{\mathrm{T}}(c) \in \mathrm{K}_{0}^{\text {split }}(\mathrm{T})$, called the index of $\mathrm{c}$ with respect to the cluster tilting subcategory T. There exists a triangle $t^{\prime} \rightarrow t \rightarrow c$ with $t, t^{\prime} \in \mathrm{T}$ (see [11, sec. 1]). The index is then defined as:

$$
\operatorname{ind}_{\mathrm{T}}(c)=[t]-\left[t^{\prime}\right],
$$

and is a well defined element of $\mathrm{K}_{0}^{\text {split }}(\mathrm{T})$.

Before giving the definition of $\alpha$ and $\beta$ from [15, def. 2.8], it remains to recall how to construct the homomorphism $\theta$ from $[15$, sec. 2.6]. We do this by following the constructions 
in [15, sec. 2.5]. Since $\mathrm{R} \subseteq \mathrm{T}$, the inclusion functor $i: \mathrm{R} \hookrightarrow \mathrm{T}$ induces the exact functor

$$
i^{*}: \operatorname{Mod} \mathrm{T} \rightarrow \operatorname{ModR}, i^{*}(F)=\left.F\right|_{\mathrm{R}},
$$

where Mod T is the abelian category of $\mathbb{C}$-linear contravariant functors $T \rightarrow \operatorname{Vect} \mathbb{C}$. Now, by [2, prop. 2.3(b)], for each $t \in$ indec T, there is a simple object $\bar{S}_{t} \in \operatorname{Mod}$ T supported at $t$, and one may see that

$$
i^{*} \bar{S}_{t}= \begin{cases}S_{t} & \text { if } t \in \text { indec R, } \\ 0 & \text { if } t \in \text { indec S }\end{cases}
$$

where $S_{t}$ denotes the simple object in Mod R supported at $t$. Due to $i^{*}$ being exact, we can restrict it to the subcategories fl T and flR, made up of the finite length objects in Mod T and Mod R, respectively. Then, there is an induced (surjective) group homomorphism

$$
\kappa: \mathrm{K}_{0}(\mathrm{flT}) \rightarrow \mathrm{K}_{0}(\mathrm{fl} \mathrm{R}),
$$

with the obvious property that

$$
\kappa\left(\left[\bar{S}_{t}\right]\right)= \begin{cases}{\left[S_{t}\right]} & \text { if } t \in \text { indec R, } \\ 0 & \text { if } t \in \text { indec S. }\end{cases}
$$

For the category Mod T, there is a functor $\bar{G}$ similar to $G$ from Eq. (2.1). It is defined by:

$$
\begin{aligned}
\bar{G}: \mathrm{C} & \rightarrow \operatorname{Mod} \mathrm{T} \\
c & \left.\mapsto \operatorname{Hom}_{\mathrm{C}}(-, \Sigma c)\right|_{\mathrm{T}} .
\end{aligned}
$$

It is not hard to see that $\bar{G}$ has the property that $i^{*} \bar{G}=G$.

We define $\theta$ to be the group homomorphism making the following diagram commute:

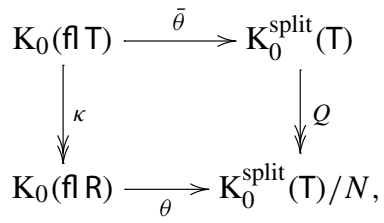

where

$$
\bar{\theta}: \mathrm{K}_{0}(\mathrm{flT}) \rightarrow \mathrm{K}_{0}^{\mathrm{split}}(\mathrm{T}), \bar{\theta}\left(\left[\bar{S}_{t}\right]\right)=[a]-\left[a^{\prime}\right],
$$

where $a, a^{\prime} \in$ add $((\operatorname{indec} \mathrm{T}) \backslash t)$ are from the exchange triangles for $t$ in (4.1).

Now, we may recall from [15, def. 2.8] that the maps $\alpha:$ obj $C \rightarrow A$ and $\beta: \mathrm{K}_{0}(\mathrm{flR}) \rightarrow A$ to a suitable ring $A$ can be defined by:

$$
\alpha(c)=\varepsilon Q\left(\operatorname{ind}_{\mathrm{T}}(c)\right) \text { and } \beta(e)=\varepsilon \theta(e),
$$

where $\varepsilon: \mathrm{K}_{0}^{\text {split }}(\mathrm{T}) / N \rightarrow A$ is a suitably chosen exponential map, meaning that:

$$
\varepsilon(0)=1, \varepsilon(a+b)=\varepsilon(a) \varepsilon(b) .
$$

Here $a$ and $b$ denote two elements of $\mathrm{K}_{0}^{\text {split }}(\mathrm{T}) / N$.

Lemma 4.1 The maps $\alpha$ and $\beta$ from (4.5) satisfy Condition F. 
Proof Consider the following triangle

$$
x \stackrel{\varphi}{\longrightarrow} y \stackrel{\omega}{\longrightarrow} z \stackrel{\psi}{\longrightarrow} \Sigma x
$$

from (3.1). Then, by definition, we have

$$
\begin{aligned}
\alpha(y) & =\varepsilon Q\left(\operatorname{ind}_{\mathrm{T}}(y)\right) \\
& \left.=\varepsilon Q \operatorname{ind}_{\mathrm{T}}(x)+\operatorname{ind}_{\mathrm{T}}(z)-\operatorname{ind}_{\mathrm{T}}(C)-\operatorname{ind}_{\mathrm{T}}\left(\Sigma^{-1} C\right)\right) \\
& =\varepsilon\left(\operatorname{ind}_{\mathrm{T}}(x)+\operatorname{ind}_{\mathrm{T}}(z)-\operatorname{ind}_{\mathrm{T}}(C)-\operatorname{ind}_{\mathrm{T}}\left(\Sigma^{-1} C\right)+N\right) \\
& =\varepsilon\left(\operatorname{ind}_{\mathrm{T}}(x)+N\right) \varepsilon\left(\operatorname{ind}_{\mathrm{T}}(z)+N\right) \varepsilon\left(-\operatorname{ind}_{\mathrm{T}}(C)-\operatorname{ind}_{\mathrm{T}}\left(\Sigma^{-1} C\right)+N\right) \\
& =(*),
\end{aligned}
$$

where $C$ in $C$ is some lifting of Coker $\bar{G}\left(\Sigma^{-1} \omega\right)$ in the sense that $\bar{G} \Sigma^{-1} C=\operatorname{Coker} \bar{G}\left(\Sigma^{-1} \omega\right)$. In the above manipulation, the second $=$ is due to [17, prop. 2.2] and the penultimate $=$ occurs since $\varepsilon$ is exponential, see Eq. (4.6).

In addition,

$$
\begin{aligned}
\alpha(x \oplus z) \beta([\operatorname{Ker} G \varphi]) & =\alpha(x) \alpha(z) \beta([\operatorname{Ker} G \varphi]) \\
& =\varepsilon Q\left(\operatorname{ind}_{\top}(x)\right) \varepsilon Q\left(\operatorname{ind}_{\top}(z)\right) \beta([\operatorname{Ker} G \varphi]) \\
& =\varepsilon\left(\operatorname{ind}_{\top}(x)+N\right) \varepsilon\left(\operatorname{ind}_{\top}(z)+N\right) \varepsilon \theta([\operatorname{Ker} G \varphi]), \\
& =(* *)
\end{aligned}
$$

where the first $=$ is due to $\alpha$ being exponential and the penultimate $=$ is just by the definition of $\beta$.

Now, using the property that $i^{*} \bar{G}=G$, it follows that

$$
[\operatorname{Ker} G \varphi]=\left[\operatorname{Ker} i^{*} \bar{G} \varphi\right] \stackrel{(1)}{=}\left[i^{*} \operatorname{Ker} \bar{G} \varphi\right] \stackrel{(2)}{=} \kappa[\operatorname{Ker} \bar{G} \varphi],
$$

where (1) follows from $i^{*}$ being an exact functor, and (2) from the definition of $\kappa$. We can now manipulate the expression $(* *)$ further:

$$
\begin{aligned}
(* *) & =\varepsilon\left(\operatorname{ind}_{\top}(x)+N\right) \varepsilon\left(\operatorname{ind}_{\top}(z)+N\right) \varepsilon \theta(\kappa([\operatorname{Ker} \bar{G} \varphi])) \\
& =\varepsilon\left(\operatorname{ind}_{\top}(x)+N\right) \varepsilon\left(\operatorname{ind}_{\top}(z)+N\right) \varepsilon Q(\bar{\theta}([\operatorname{Ker} \bar{G} \varphi])) \\
& =\varepsilon\left(\operatorname{ind}_{\top}(x)+N\right) \varepsilon\left(\operatorname{ind}_{\top}(z)+N\right) \varepsilon(\bar{\theta}([\operatorname{Ker} \bar{G} \varphi])+N) \\
& =(* * *),
\end{aligned}
$$

where the second equality is due to the commutativity of Diagram (4.4).

Comparing $(*)$ to $(* * *)$, we see that the required equality for Condition $\mathrm{F}$ is satisfied if

$$
\bar{\theta}([\operatorname{Ker} \bar{G} \varphi])=-\left(\operatorname{ind}_{\mathrm{T}}(C)+\operatorname{ind}_{\mathrm{T}}\left(\Sigma^{-1} C\right)\right) .
$$

Making use of the "rolling" property on our triangle in (4.7), we obtain the following sequence:

$$
\Sigma^{-1} y \stackrel{\Sigma^{-1} \omega}{\longrightarrow} \Sigma^{-1} z \stackrel{\Sigma^{-1} \psi}{\longrightarrow} x \stackrel{\varphi}{\longrightarrow} y \stackrel{\omega}{\longrightarrow} z
$$

where any four consecutive terms form a triangle. Furthermore, since $\bar{G}$ is a homological functor, we may apply it to this sequence and produce the following long exact sequence in flT:

$$
\bar{G} \Sigma^{-1} y \stackrel{\bar{G} \Sigma^{-1} \omega}{\longrightarrow} \bar{G} \Sigma^{-1} z \stackrel{\bar{G} \Sigma^{-1} \psi}{\longrightarrow} \bar{G} x \stackrel{\bar{G} \varphi}{\longrightarrow} \bar{G} y \stackrel{\bar{G} \omega}{\longrightarrow} \bar{G} z
$$


This shows $\operatorname{Coker} \bar{G} \Sigma^{-1} \omega=\operatorname{Ker} \bar{G} \varphi$. Moreover, $C$ is chosen such that $\bar{G} \Sigma^{-1} C=$ Coker $\bar{G} \Sigma^{-1} \omega$, and hence $\operatorname{Ker} \bar{G} \varphi=\bar{G} \Sigma^{-1} C$. We can hence compute as follows:

$$
\begin{aligned}
\bar{\theta}([\operatorname{Ker} \bar{G} \varphi]) & =\bar{\theta}\left(\left[\bar{G} \Sigma^{-1} C\right]\right) \\
& =-\left(\operatorname{ind}_{\mathrm{T}}\left(\Sigma^{-1} C\right)+\operatorname{ind}_{\mathrm{T}}\left(\Sigma\left(\Sigma^{-1} C\right)\right)\right) \\
& =-\left(\operatorname{ind}_{\mathrm{T}}(C)+\operatorname{ind}_{\mathrm{T}}\left(\Sigma^{-1} C\right)\right),
\end{aligned}
$$

where the second $=$ is due to [15, lem. 2.10]. We can now see that Eq. (4.8) holds, and hence the lemma is proved.

Remark 4.2 Through Lemma 4.1 and Theorem 3.2, we have managed to recover [15, thm. 2.11]. Indeed, [15, thm. 2.11] states that when

$$
\Delta=\Sigma c \rightarrow b \rightarrow c
$$

is an AR triangle in C such that $\bar{G} c$ and $\bar{G}(\Sigma c)$ have finite length in Mod T, the maps $\alpha$ and $\beta$ from Eq. (4.5) satisfy the frieze-like condition given in [15, def. 1.4]. By Lemma 4.1, we know that $\alpha$ and $\beta$ as defined in Eq. (4.5) satisfy Condition F. Theorem 3.2 proves that any $\alpha$ and $\beta$ satisfying Condition $\mathrm{F}$ also satisfy the frieze-like condition for $\Delta$ (recovering [15, thm. 2.11]). Hence by [15, thm. 1.6], these $\alpha$ and $\beta$ turn $\rho$ into a generalised frieze.

\section{The Multiplication Formula from [14]}

In this section we demonstrate some of the technicalities behind the proof of the multiplication formula for $\pi$ from Eq. (1.7), proved in [14, prop. 4.4]. This is done with a view of proving a similar formula for $\rho$ in Sect. 6. Following the setup of [14, sec. 4], for this section we do not require a cluster tilting subcategory $\mathrm{T}$, as the theory in [14] uses only the rigid subcategory $\mathrm{R}$.

Let $m \in \operatorname{indec} C$ and $r \in$ indec $\mathrm{R}$ be indecomposable objects such that $\operatorname{Ext}_{\mathrm{C}}^{1}(r, m)$ and $\operatorname{Ext}_{C}^{1}(m, r)$ both have dimension one over $\mathbb{C}$. As in [14, rem. 4.2], this allows us to construct the following nonsplit triangles in $\mathrm{C}$ :

$$
m \stackrel{\mu}{\longrightarrow} a \stackrel{\gamma}{\longrightarrow} r \stackrel{\delta}{\longrightarrow} \Sigma m
$$

and

$$
r \stackrel{\sigma}{\longrightarrow} b \stackrel{\eta}{\longrightarrow} m \stackrel{\zeta}{\longrightarrow} \Sigma r,
$$

with $\delta$ and $\zeta$ nonzero. Note that "rolling" the first triangle gives:

$$
\Sigma^{-1} r \stackrel{-\Sigma^{-1} \delta}{\longrightarrow} m \stackrel{\mu}{\longrightarrow} a \stackrel{\gamma}{\longrightarrow} r,
$$

which is also a triangle in C. Applying the functor $G$ to both the "rolled" triangle and the triangle in (5.2) gives the following exact sequences in Mod R, obtained in [14]:

$$
G\left(\Sigma^{-1} r\right) \stackrel{-G\left(\Sigma^{-1} \delta\right)}{\longrightarrow} G m \stackrel{G \mu}{\longrightarrow} G a \longrightarrow 0
$$

and

$$
0 \longrightarrow G b \stackrel{G \eta}{\longrightarrow} G m \stackrel{G \zeta}{\longrightarrow} G(\Sigma r) .
$$


Remark 5.1 (1) The zeros arise in each exact sequence due to $G(r)=\left.\operatorname{Hom}(-, \Sigma r)\right|_{\mathrm{R}}$ being the zero functor. Indeed, since $\mathrm{R}$ is rigid, evaluating $G(r)$ at any $x$ in $\mathrm{R}$ will make the corresponding Hom-space zero.

(2) The exact sequences are in Mod R; that is, each term is a $\mathbb{C}$-linear contravariant functor $\mathrm{R} \rightarrow$ Vect $\mathbb{C}$

Letting Gr denote the Grassmannian of submodules, we have morphisms of algebraic varietes,

$$
\begin{aligned}
\operatorname{Gr}(G a) \stackrel{\xi}{\longrightarrow} & \operatorname{Gr}(G m) \stackrel{v}{\longleftarrow} \operatorname{Gr}(G b), \\
P \longmapsto & (G \mu)^{-1}(P), \\
& (G \eta)(N) \longleftrightarrow \mid N .
\end{aligned}
$$

It was proved in [14, Lemma 4.3] that if $M$ in Mod R is some subfunctor of $G m$, then either $M \subseteq \operatorname{Im} G \eta$ or $\operatorname{Ker} G \mu \subseteq M$, but not both. This means that for $M \subseteq G m$ we can find either a subfunctor $N \subseteq G b$ such that $(G \eta)(N)=M$ or we can find $P \subseteq G a$ such that $(G \mu)^{-1}(P)=M$. Hence, the subfunctor $M$ is either of the form $(G \eta)(N)$ or $(G \mu)^{-1}(P)$.

It is hence clear that $\operatorname{Gr}(\mathrm{Gm})$ is isomorphic to the disjoint union of the images of $\xi$ and $v$. That is,

$$
\operatorname{Gr}(G m) \cong \operatorname{Gr}(G b) \bigsqcup \operatorname{Gr}(G a)
$$

Remark 5.2 (1) We should note that for $M$ in Mod R, the Grassmannian $\operatorname{Gr}(M)$ is an algebraic variety. Therefore, it makes sense to calculate the Euler characteristic of $\operatorname{Gr}(G m), \operatorname{Gr}(G a)$ and $\operatorname{Gr}(G b)$.

(2) In addition, we note that $\xi$ and $v$ are both constructible maps, hence the images of $\xi$ and $v$ form constructible subsets in $\operatorname{Gr}(G m)$. See [17, Section 2.1] for the definitions of a constructible map and a constructible set.

The following statement then follows in [14]:

$$
\chi(\operatorname{Gr}(G m))=\chi(\operatorname{Gr}(G b))+\chi(\operatorname{Gr}(G a)),
$$

where $\chi$ again denotes the Euler characteristic defined by cohomology with compact support (see [13, p. 93]). Using Remark 5.2, since the images of $\xi$ and $v$ are constructible sets inside $\operatorname{Gr}(G m)$, we know that $\chi$ is additive (see [13, p. 92, item (3)]), which gives the above equality in (5.3). That is

$$
\pi(m)=\pi(a)+\pi(b) .
$$

\section{Adaptation of the Multiplication Formula to [15]}

This section builds on the material covered in the previous section and makes necessary adjustments and additions in order to obtain the multiplication formula for $\rho$, given in Theorem 6.2. Clearly, now that we are back working with $\rho$, we again require the setup of Sect. 2; that is, we need a cluster tilting subcategory $\mathrm{T}$, with $\mathrm{R} \subseteq \mathrm{T}$.

As with $\pi$, we look to understand how to evaluate $\rho$ for some $m \in$ indec $C$. In the definition of $\rho$, we take a sum over $e \in \mathrm{K}_{0}(f \mathrm{R})$. In order to do this, we will require knowledge of the Grothendieck group $\mathrm{K}_{0}(\mathrm{fl} \mathrm{R})$ and the $\mathrm{K}_{0}$-classes of some of its key elements. 
Firstly, we know

$$
[v N]=[N] .
$$

Indeed, by definition, $v N=(G \eta)(N)$, and since $G \eta$ is injective, $(G \eta)(N)$ and $N$ have the same composition series. Hence, the above equality is true.

To find $[\xi P]$, we first note that by definition, $\xi P=(G \mu)^{-1} P$, and therefore, $[\xi P]=$ $\left[(G \mu)^{-1} P\right]$. A consequence of the Second Isomorphism Theorem is that a composition series of $(G \mu)^{-1} P$ can be obtained by concatenating composition series of $P$ and of $\operatorname{Ker} G \mu$. That is, $\left[(G \mu)^{-1}(P)\right]=[P]+[\operatorname{Ker} G \mu]$. So, the $\mathrm{K}_{0}$-classes are:

$$
[v N]=[N] \text { and }[\xi P]=[P]+[\operatorname{Ker} G \mu] .
$$

Now that we have some useful information about the $\mathrm{K}_{0}$-classes, we can take a more in depth look at $\rho$. Consider $r$ in R, and let us calculate $\rho(r)$ :

$$
\begin{aligned}
\rho(r) & =\alpha(r) \sum_{e} \chi\left(\operatorname{Gr}_{e}(G(r))\right) \beta(e) \\
& =\alpha(r) \sum_{e} \chi\left(\operatorname{Gr}_{e}(0)\right) \beta(e) \\
& =\alpha(r) \beta(0) \\
& =\alpha(r) .
\end{aligned}
$$

In the above calculation, the third $=$ is due to $\chi\left(\mathrm{Gr}_{e}(0)\right)$ being zero for all nonzero $e \in \mathrm{K}_{0}(\mathrm{fl} \mathrm{R})$ and one when $e=0$. The last $=$ is since $\beta$ is exponential.

Now consider $\rho(r) \rho(m)$ for $m \in$ indec $\mathrm{C}$ :

$$
\begin{aligned}
\rho(r) \rho(m) & =\alpha(r) \alpha(m) \sum_{e} \chi\left(\operatorname{Gr}_{e}(G m)\right) \beta(e) \\
& =\alpha(r) \alpha(m)\left(\sum_{e} \chi\left(\operatorname{Im} \xi \cap \operatorname{Gr}_{e}(G m)\right)+\chi\left(\operatorname{Im} v \cap \operatorname{Gr}_{e}(G m)\right)\right) \beta(e),
\end{aligned}
$$

where the second equality arises from $\operatorname{Gr}(G m)$ being the disjoint union of the images of $\xi$ and $v$. We now make an important remark about the two intersections in the second equality above.

Remark 6.1 (1) The first intersection is given by the image of $\xi$ when applied to $\operatorname{Gr}_{e}(G a)$.

Indeed,

$$
\begin{aligned}
\operatorname{Im} \xi \cap \mathrm{Gr}_{e}(G m) & =\{\xi P \mid[\xi P]=e\} \\
& =\{\xi P \mid[P]=e-[\operatorname{Ker} G \mu]\} \\
& =\xi\left(\operatorname{Gr}_{e-[\operatorname{Ker} G \mu]}(G a)\right) .
\end{aligned}
$$

Here we used the fact that $[\xi P]=[P]+[\operatorname{Ker} G \mu]$.

(2) The second intersection can be obtained in a similar way:

$$
\begin{aligned}
\operatorname{Im} v \cap \mathrm{Gr}_{e}(G m) & =\{v N \mid[v N]=e\} \\
& =\{v N \mid[N]=e\} \\
& =v\left(\operatorname{Gr}_{e}(G b)\right)
\end{aligned}
$$


Using this remark, we can continue to calculate $\rho(r) \rho(m)$, obtaining:

$$
\begin{aligned}
\rho(r) \rho(m) & =\alpha(r) \alpha(m) \sum_{e}\left(\chi\left(\xi\left(\operatorname{Gr}_{e-[\operatorname{Ker} G \mu]}(G a)\right)+\chi\left(v\left(\operatorname{Gr}_{e}(G b)\right)\right)\right) \beta(e)\right. \\
& =\alpha(r) \alpha(m) \sum_{e}\left(\chi\left(\operatorname{Gr}_{e-[\operatorname{Ker} G \mu]}(G a)\right)+\chi\left(\operatorname{Gr}_{e}(G b)\right)\right) \beta(e) .
\end{aligned}
$$

We can discard $\xi$ and $v$ in the final expression since they are both embeddings.

Theorem 6.2 Let $m \in \operatorname{indec} C$ and $r \in \operatorname{indec} R$ such that $\operatorname{Ext}_{C}^{1}(r, m)$ and $\operatorname{Ext}_{C}^{1}(m, r)$ both have dimension one over $\mathbb{C}$. Then, there are nonsplit triangles

$$
m \stackrel{\mu}{\longrightarrow} a \stackrel{\gamma}{\longrightarrow} r \stackrel{\delta}{\longrightarrow} \Sigma m \quad \text { and } \quad r \stackrel{\sigma}{\longrightarrow} b \stackrel{\eta}{\longrightarrow} m \stackrel{\zeta}{\longrightarrow} \Sigma r,
$$

with $\delta$ and $\zeta$ nonzero. Let Gm have finite length in Mod $R$, then

$$
\rho(r) \rho(m)=\rho(a)+\rho(b) .
$$

Proof We first note that since $G m$ has finite length, then so do $G a$ and $G b$. This follows immediately from [14, rem. 4.2].

Now, by making the substitution $f=e-[\operatorname{Ker} G \mu]$ in Eq. (6.1), observe that

$$
\begin{gathered}
\rho(r) \rho(m)=\alpha(r) \alpha(m) \sum_{f} \chi\left(\operatorname{Gr}_{f}(G a)\right) \beta(f+[\operatorname{Ker} G \mu]) \\
+\alpha(r) \alpha(m) \sum_{e} \chi\left(\operatorname{Gr}_{e}(G b)\right) \beta(e) \\
\stackrel{(a)}{=} \alpha(r) \alpha(m) \sum_{f} \chi\left(\operatorname{Gr}_{f}(G a)\right) \beta(f) \beta([\operatorname{Ker} G \mu]) \\
+\alpha(r) \alpha(m) \sum_{e} \chi\left(\operatorname{Gr}_{e}(G b)\right) \beta(e) \\
\stackrel{(b)}{=} \alpha(r) \alpha(m) \beta([\operatorname{Ker} G \mu]) \sum_{f} \chi\left(\operatorname{Gr}_{f}(G a)\right) \beta(f) \\
+\alpha(r) \alpha(m) \sum_{e} \chi\left(\operatorname{Gr}_{e}(G b)\right) \beta(e) .
\end{gathered}
$$

Here (a) is due to $\beta$ being exponential (see [15, setup 1.1]) and (b) is due to $\beta$ ([Ker $G \mu]$ ) being a constant.

Now, consider $\operatorname{Ker} G \sigma$. Since $G(r)=0$, then $\operatorname{Ker} G \sigma=0$, and clearly $[\operatorname{Ker} G \sigma]=0$. The map $\beta$ is exponential, and therefore $\beta([\operatorname{Ker} G \sigma])=\beta(0)=1$. We can insert this in to Eq. (6.2) and see that:

$$
\begin{aligned}
& \rho(r) \rho(m)=\alpha(r) \alpha(m) \beta([\operatorname{Ker} G \mu]) \sum_{f} \chi\left(\operatorname{Gr}_{f}(G a)\right) \beta(f) \\
& +\alpha(r) \alpha(m) \beta([\operatorname{Ker} G \sigma]) \sum_{e} \chi\left(\operatorname{Gr}_{e}(G b)\right) \beta(e) .
\end{aligned}
$$

Applying Condition $\mathrm{F}$ to our two triangles in the theorem, whilst remembering that $\alpha$ is exponential, gives

$$
\begin{aligned}
& \alpha(a)=\alpha(r) \alpha(m) \beta([\operatorname{Ker} G \mu]) \\
& \alpha(b)=\alpha(r) \alpha(m) \beta([\operatorname{Ker} G \sigma]) .
\end{aligned}
$$


Returning these equalities into Eq. (6.3), the expression for $\rho(r) \rho(m)$ becomes:

$$
\begin{aligned}
\rho(r) \rho(m) & =\alpha(a) \sum_{f} \chi\left(\operatorname{Gr}_{f}(G a)\right) \beta(f)+\alpha(b) \sum_{e}\left(\operatorname{Gr}_{e}(G b)\right) \beta(e) \\
& =\rho(a)+\rho(b) .
\end{aligned}
$$

\section{Example for $\mathrm{C}\left(\boldsymbol{A}_{5}\right)$}

In this section, we will demonstrate the multiplication formula for $\rho$ in Theorem 6.2 by recomputing a vertex in the AR quiver in Fig. 3. We first give some brief background on the polygon model for $\mathrm{C}=\mathrm{C}\left(A_{n}\right)$, the cluster category of Dynkin type $A_{n}$. Here, $n \geq 2$ is an integer. By [7], the indecomposables of $C$ can be identified with the diagonals of a regular $(n+3)$-gon $P$ with the set of vertices $\{0, \ldots, n+2\}$. By [14, thm. 5.4], the indecomposables of the rigid subcategory $\mathrm{R} \subseteq \mathrm{T}$ give a polygon dissection of $P$, and by [4] the cluster tilting subcategory T gives a full triangluation of the $(n+3)$-gon. Indeed, recall that there is a full subcategory $\mathrm{S}$, which is closed under direct sums and summands, such that

$$
\text { indec } \mathrm{T}=\text { indec } \mathrm{R} \cup \text { indec } \mathrm{S} \text {. }
$$

Hence, the indecomposables in S correspond to a triangulation of each of the cells of $P$ given by the polygon dissection from indec $\mathrm{R}$. We should note in addition that each edge of the $(n+3)$-gon is identified with the zero object inside $C$.

This model also comes with the convenient property that for two indecomposables $a$ and $b$ in $\mathrm{C}$

$$
\operatorname{dim}_{\mathbb{C}} \operatorname{Ext}_{C}^{1}(a, b)=\left\{\begin{array}{l}
1, \text { if } a \text { and } b \text { cross } \\
0, \text { otherwise. }
\end{array}\right.
$$

It is known by Theorem 6.2 that for $m \in \operatorname{indec} C$ and $r \in \operatorname{indec} \mathrm{R}$ such that $\operatorname{dim}_{\mathbb{C}} \operatorname{Ext}_{C}^{1}(m, r)=$ $\operatorname{dim}_{\mathbb{C}} \operatorname{Ext}_{C}^{1}(r, m)=1$, then

$$
\rho(r) \rho(m)=\rho(a)+\rho(b),
$$

where $a$ and $b$ are the middle terms of the nonsplit extensions in (5.1) and (5.2). In the case of $\mathrm{C}=\mathrm{C}\left(A_{n}\right), a$ and $b$ can be obtained as seen in the polygon in Fig. 4, where we have $a=a_{1} \oplus a_{2}$ and $b=b_{1} \oplus b_{2}$. See [15, sec. 5] for details.

Now, to compute our example, we refer to the setup of $[15$, sec. 3$]$; that is, we set $\mathrm{C}=$ $\mathrm{C}\left(A_{5}\right)$, the cluster category of Dynkin type $A_{5}$. Thus, the indecomposables of $C$ can be identified with the diagonals on a regular 8-gon. As in [15] we will denote by $\{a, b\}$ the indecomposable corresponding to the diagonal connecting the vertices $a$ and $b$. We use the same polygon triangulation as in [15, sec. 3]; that is, indec R corresponds to the dotted diagonals in Fig. 5 and indec $\mathrm{S}$ corresponds to the dashed diagonals. Hence, R contains the following indecomposable objects :

$$
\{2,5\},\{2,7\},
$$

whilst the indecomposables in $\mathrm{S}$ are:

$$
\{1,7\},\{2,4\},\{5,7\} .
$$




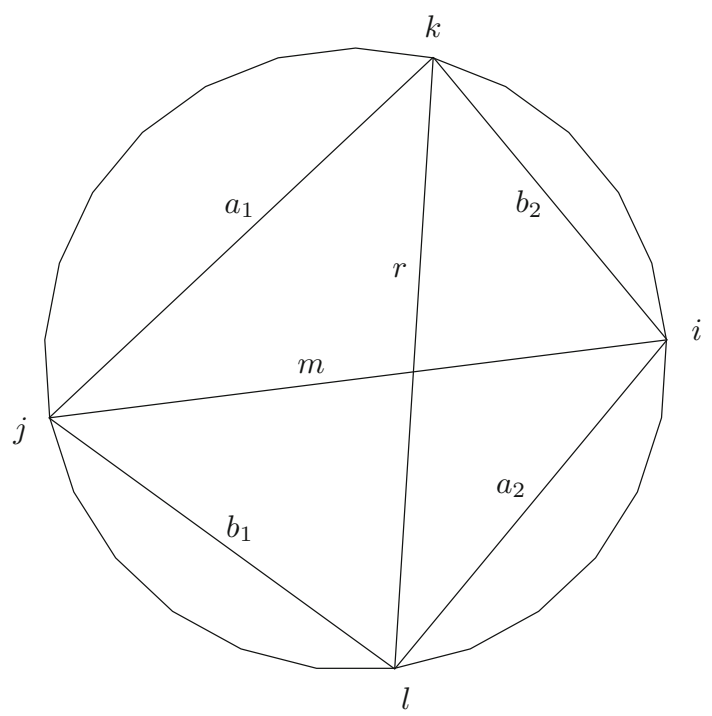

Fig. 4 There are nonsplit extensions $m \rightarrow a_{1} \oplus a_{2} \rightarrow r$ and $r \rightarrow b_{1} \oplus b_{2} \rightarrow m$

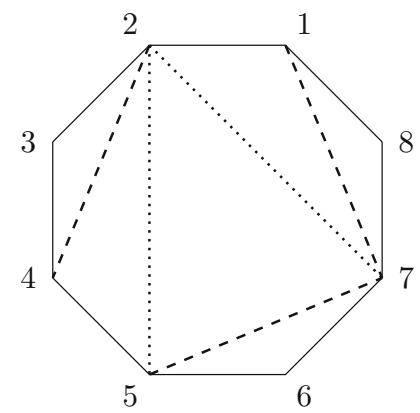

Fig. 5 Dotted diagonals correspond to indecomposables in indec $\mathrm{R}$ and dashed diagonals correspond to indecomposables in indec $\mathrm{S}$

These indecomposables in S fit in the following exchange triangles:
$\{1,7\}$
$\{2,7\} \longrightarrow\{2,8\}$
$\{2,8\}$
0
$\{1,7\}$
$\{2,4\}$
$\{2,5\}$
$\{3,5\}$
$\{3,5\}$
0
$\{5,7\}$
$\{2,5\} \longrightarrow\{2,6\}$
$\{2,6\}$
$\{2,7\}$ $\{5,7\}$.

Then, applying the definition of $N$ from (4.3), it is easily seen that

$$
N=\langle[2,5],[2,7]\rangle,
$$

Here, we denote by $[a, b]$ the $\mathrm{K}_{0}^{\text {split }}$-class of the indecomposable $\{a, b\}$. We also have

$$
\mathrm{K}_{0}^{\text {split }}(\mathrm{T}) / N=\langle[1,7]+N,[2,4]+N,[5,7]+N\rangle,
$$


and let the exponential map $\varepsilon: \mathrm{K}_{0}^{\text {split }}(\mathrm{T}) / N \rightarrow \mathbb{Z}\left[u^{ \pm 1}, v^{ \pm 1}, z^{ \pm 1}\right]$ be given by:

$$
\varepsilon([1,7]+N)=u, \varepsilon([2,4]+N)=v, \varepsilon([5,7]+N)=z .
$$

We will now demonstrate how to calculate $\rho(\{4,6\})$ using an alternative method to that in [15, ex. 3.5]. We will compute it by applying the multiplication formula for $\rho$ in Theorem 6.2. Since $\operatorname{dim}_{\mathbb{C}} \operatorname{Ext}^{1}(\{4,6\},\{2,5\})=\operatorname{dim}_{\mathbb{C}} \operatorname{Ext}^{1}(\{2,5\},\{4,6\})=1$, we may set $r=\{2,5\}$, and using Fig. 4 , we know that $\{4,6\}$ sits in the following nonsplit extensions:

$$
\{4,6\} \rightarrow\{2,4\} \rightarrow\{2,5\},\{2,5\} \rightarrow\{2,6\} \rightarrow\{4,6\} .
$$

Applying Theorem 6.2, we get the following equality:

$$
\rho(\{2,5\}) \rho(\{4,6\})=\rho(\{2,4\})+\rho(\{2,6\}) .
$$

Due to the fact that the diagonals corresponding to $\{2,5\},\{2,4\}$ and $\{2,6\}$ do not cross any diagonals in indec $\mathrm{R}$, it is immediate that

$$
G(\{2,5\})=G(\{2,4\})=G(\{2,6\})=0 .
$$

Hence, by the definition of $\rho$, Eq. (7.2) becomes:

$$
\alpha(\{2,5\}) \rho(\{4,6\})=\alpha(\{2,4\})+\alpha(\{2,6\}) .
$$

In order to calculate $\alpha$ of each of the indecomposables $\{2,5\},\{2,4\}$ and $\{2,6\}$, we first calculate their indices with respect to $T$.

$\{2,5\}$ sits in the following triangle:

$$
0 \rightarrow\{2,5\} \rightarrow\{2,5\}
$$

and since $\{2,5\} \in$ indec $T$, we see that:

$$
\operatorname{ind}_{\top}(\{2,5\})=[2,5] .
$$

By the same logic,

$$
\operatorname{ind}_{\top}(\{2,4\})=[2,4] \text {. }
$$

We note that one of the exchange triangles for $\{2,6\}$ is:

$$
\{5,7\} \rightarrow\{2,5\} \rightarrow\{2,6\},
$$

and hence

$$
\operatorname{ind}_{T}(\{2,6\})=[2,5]-[5,7] .
$$

Since $[2,5] \in N$, using the definition of $\alpha$ from [15, def. 2.8], we see that:

$$
\begin{aligned}
\alpha(\{2,5\}) & =\varepsilon Q\left(\operatorname{ind}_{\mathrm{T}}(\{2,5\})\right. \\
& =\varepsilon Q([2,5]) \\
& =\varepsilon([2,5]+N) \\
& =\varepsilon(0) \\
& =1
\end{aligned}
$$


and hence, the right hand side of Eq. (7.3) becomes $\alpha(\{2,5\}) \rho(\{4,6\})=\rho(\{4,6\})$. Substituting back into Eq. (7.3), we obtain:

$$
\begin{aligned}
\rho(\{4,6\}) & =\alpha(\{2,4\})+\alpha(\{2,6\}) \\
& =\varepsilon Q\left(\operatorname{ind}_{\mathrm{T}}(\{2,4\})\right)+\varepsilon Q\left(\operatorname{ind}_{\mathrm{T}}(\{2,6\})\right) \\
& \stackrel{(1)}{=} \varepsilon Q([2,4])+\varepsilon Q([2,5]-[5,7]) \\
& =\varepsilon([2,4]+N)+\varepsilon(-[5,7]+N) \\
& \stackrel{(2)}{=} v+z^{-1} \\
& =\frac{1+v z}{z},
\end{aligned}
$$

where (1) is by substituting the values from Eqs. (7.5) and (7.6), and (2) is due to the definition of $\varepsilon$ from Eq. (7.1). We notice here that this is indeed the same result for $\rho(\{4,6\})$ obtained in [15, ex. 3.5].

Similar computations for the other indecomposables in $\mathrm{C}\left(A_{5}\right)$ will produce the generalised frieze as drawn in Fig. 3 in the introduction.

Remark 7.1 In general, the formula from Theorem 6.2 can be applied iteratively to calculate $\rho(m)$. Indeed,

$$
\rho(r) \rho(m)=\rho(a)+\rho(b)
$$

is an iterative formula on $m$, and hence, calculating $\rho$ of each indecomposable in C can be reduced to calculating $\rho$ of the indecomposables in $C$ whose corresponding diagonals in the $(n+3)$-gon do not cross any of the diagonals in R. Namely, it is clear from Fig. 4 that each of $a_{1}, a_{2}, b_{1}$ and $b_{2}$ sit inside "smaller" polygons than $m$. Here, the smaller polygons are those obtained from $r$ dissecting the $(\mathrm{n}+3)$-gon. Since $\mathrm{R}$ consists of only non-crossing diagonals, the remaining diagonals in $\mathrm{R}$ sit inside these smaller polygons. Reapplying Theorem 6.2 to each of $a_{1}, a_{2}, b_{1}$ and $b_{2}$ will again create a series of even smaller polygons, containing a new $a$ or $b$. After repeated iterations, this process will eventually terminate at the stage when the new $a$ or $b$ does not cross any of the diagonals in R.

Now, in the case when a diagonal, say $m^{\prime}$, does not cross a diagonal in R, calculating $\rho\left(\mathrm{m}^{\prime}\right)$ is acheived by calculating $\alpha\left(m^{\prime}\right)$. This is clear since $G\left(m^{\prime}\right)=0$. Computing $\alpha\left(m^{\prime}\right)$ is done by calculating the index of $m^{\prime}$, and then applying the maps $Q$ and $\varepsilon$. Hence, finding $\rho(m)$ for each $m \in$ indec $C$ can be reduced by Theorem 6.2 to computing the index of each of the indecomposables in $\mathrm{C}$ whose corresponding diagonals do not cross any diagonals in $\mathrm{R}$.

Acknowledgements The author acknowledges economic support in the form of a studentship from the School of Mathematics and Statistics at Newcastle University.

Open Access This article is distributed under the terms of the Creative Commons Attribution 4.0 International License (http://creativecommons.org/licenses/by/4.0/), which permits unrestricted use, distribution, and reproduction in any medium, provided you give appropriate credit to the original author(s) and the source, provide a link to the Creative Commons license, and indicate if changes were made.

\section{References}

1. Assem, I., Dupont, G.: Friezes and a construction of the Euclidean cluster variables. J. Pure Appl. Algebra 215, 2322-2340 (2011). https://doi.org/10.1016/j.jpaa.2012.12.013

2. Auslander, M.: Representation theory of Artin algebras II. Commun. Algebra 1, 269-310 (1974) 
3. Buan, A.B., Iyama, O., Reiten, I., Scott, J.: Cluster structure for 2-Calabi-Yau categories and unipotent groups. Compos. Math. 145, 1035-1079 (2009)

4. Buan, A.B., Marsh, R., Reineke, M., Reiten, I., Todorov, G.: Tilting theory and cluster combinatorics. Adv. Math. 204, 572-618 (2006)

5. Brüstle, T., Zhang, J.: On the cluster category of a marked surface without punctures. Algebra Number Theory 5(4), 529-566 (2011)

6. Caldero, P., Chapoton, F.: Cluster algebras as Hall algebras of quiver representations. Comment. Math. Helv. 81, 595-616 (2006). https://doi.org/10.4171/CMH/65

7. Caldero, P., Chapoton, F., Schiffler, R.: Quivers with relations arising from clusters $\left(A_{n}\right.$ case). Trans. Am. Math. Soc. 358, 1347-1364 (2006)

8. Caldero, P., Keller, B.: From triangulated categories to cluster algebras. Invent. Math. 172, 169-211 (2008)

9. Conway, J.H., Coxeter, H.S.M.: Triangulated polygons and frieze patterns. Math. Gaz. 57(400), 87-94 (1973)

10. Conway, J.H., Coxeter, H.S.M.: Triangulated polygons and frieze patterns. Math. Gaz. 57(401), 175-183 (1973)

11. Dehy, R., Keller, B.: On the combinatorics of rigid objects in 2-Calabi-Yau categories. Int. Math. Res. Not. (11), (2008)

12. Domínguez, S., Geiss, C.: A Caldero-Chapoton formula for generalized cluster categories. J. Algebra 399, 887-893 (2014). https://doi.org/10.1016/j.jalgebra.2013.10.018

13. Fulton, W.: Introduction to Toric Varieties. Annals of Mathematics Studies, vol. 131. Princeton University Press, Princeton (1993)

14. Holm, T., Jørgensen, P.: Generalized friezes and a modified Caldero-Chapoton map depending on a rigid object. Nagoya Math. J. 218, 101-124 (2015)

15. Holm, T., Jørgensen, P.: Generalized friezes and a modified Caldero-Chapoton map depending on a rigid object II. Bull. Sci. Math. 140, 112-131 (2016)

16. Marsh, R., Reineke, M., Zelevinsky, A.: Generalized associahedra via quiver representations. Trans. Am. Math. Soc. 355(10), 4171-4186 (2003)

17. Palu, Y.: Cluster characters, II: a multiplication formula. Proc. Lond. Math. Soc. 3(104), 57-78 (2012) 